\title{
On the critical dynamics of ferromagnets
}

\section{E. Frey and F. Schwabl}

Institut für Theoretische Physik, Physik-Department der Technischen Universität München, Garching, Federal Republic of Germany

Z. Phys. B - Condensed Matter 71, 355-368 (1988)

The paper contains several misprints and a missing factor $\frac{1}{2}$ in some of the intermediate equations. None of the final results and conclusions are changed.

Eqs. (3.5a) and (3.5b): Replace 4 by 2 .

Eqs. (3.8a) and (3.8b): Replace $\pi^{2}$ by $2 \pi^{2}$.

Eq. (3.13):

Eq. (3.17):

Replace $2 \pi^{4}$ by $4 \pi^{4}$ and $4 \pi^{4}$ by $8 \pi^{4}$.

Eq. (3.21):

Delete 4 .

Eq. $(3.25 \mathrm{a})$ :

Replace 8 by 4 .

Eq. (3.29)

Replace $\int \mathrm{d} t$ by $\int \frac{\mathrm{d} \omega}{2 \pi}$.

Should read $H=\frac{\left(k_{B} T\right)^{2}}{32 \pi^{6}\left(\Lambda a^{-5 / 2}\right)^{3}}\left(q_{D} a\right)^{-3 / 2}$ for bcc Fe.

In the first paragraph after Eq. (3.23) $B_{\mathrm{th}}$ and $B_{\text {exp }}$ should be replaced by $B_{\mathrm{th}}=151 \mu \mathrm{eV}$ and $B_{\text {exp }}=123 \mu \mathrm{eV}$ (see also Kötzler, J.: Phys. Rev. B38, 12027 (1988)).

In the second paragraph after Eq. $(3.31 \mathrm{~b}) H_{\text {th }}$ and $q_{D}$ should be replaced by $H_{\text {th }}$ $=11.0 \cdot 10^{-13} \mathrm{~s}$ and $q_{D}=0.045 \AA^{-1}$. If one inserts in Eq. (3.29) the experimental value $A=130 / 5.1326 \mathrm{meV} \AA^{5 / 2}$, which is very close to the solution of the complete mode coupling equations (Frey, E., Schwabl, F., Thoma, S.: Phys. Lett. A 129, 343 (1988)), one finds $H_{\mathrm{th}}=6.16 \cdot 10^{-13} \mathrm{~s}$.

E. Frey, F. Schwab]

Institut für Theoretische Physik

Physik-Department

Technische Universität München

James-Franck-Strasse

D-8046 Garching bei München

Federal Republic of Germany 